\title{
Die Gamma-GT ist sehr unzuverlässig
}

— Eine Erhöhung der Gamma-GT wird oft als wichtigster Hinweis auf eine alkoholische Leberschädigung interpretiert. "Doch die Korrelation zwischen der Gamma-GT und dem Alkohol ist sehr schlecht", sagte Prof. Ulrich Treichel, Hagenow. Jeder zweite Patient mit einer Gamma-GT-Erhöhung hat nach seinen Worten gar kein Alkoholproblem. Auch bestehe keine Korrelation zwischen Alkoholmenge und der
Höhe der Gamma-GT. Nach Erreichen einer dauerhaften Karenz bleibe die Gamma-GT bei 30\% dieser Patienten weiter erhöht. Grundsätzlich kann ein Alkoholabusus mit Laborwerten allein nie eindeutig nachgewiesen oder ausgeschlossen werden, so Treichel, denn der Fokus der Alkoholkrankheit sitzt nicht in der Leber. Sie muss es nur ausbaden!

STI = Internistenkongress 2012, Wiesbaden, 14.-17.4.2012

\section{MAMMAKARZINOMPRÄVENTION}

\section{Folsäure hält Brustveränderung durch Alkohol nicht auf}

— Mit Folsäure gelingt es nicht, die durch Alkoholkonsum in jungen Jahren angestoßenen gutartigen Brustveränderungen bei Frauen zu verhindern.

Bereits vor etwa einer Dekade belegten US-Wissenschaftler, dass junge Frauen, die häufig Alkohol trinken, ein deutlich erhöhtes Risiko für gutartige Brustveränderungen haben. Einige dieser Veränderungen sind mit einem erhöhten Brustkrebsrisiko assoziiert. Schon seit längerem gilt Alkohol als Risikofaktor für Brustkrebs. Andererseits ist Folsäure für die Genexpression und die Reparatur von DNA-Schäden wichtig und wird durch Alkoholkonsum verbraucht. Deshalb hofften viele Frauen, den schädlichen Effekt von Alkohol auf das Brustgewebe zu verhindern, indem sie mehr Folsäure aufnahmen. Blatt- und Kohlgemüse, Tomaten, Orangen und Getreide, aber auch Leber und Niere sind gute Folsäurelieferanten.

Dr. Ying Liu et al. aus St. Louis (USA) werteten nun die Angaben von mehr als 29000 Frauen aus der Nurses' Health Study II aus. Die Frauen hatten 1989 Auskunft über ihren Alkoholkonsum als Heranwachsende gegeben sowie Fragen zu Ernährungsgewohnheiten in diesem Alter beantwortet. Zwischen 1991 und 2001 wurden bei 659 Frauen proliferative gutartige Brusterkrankungen diagnostiziert.

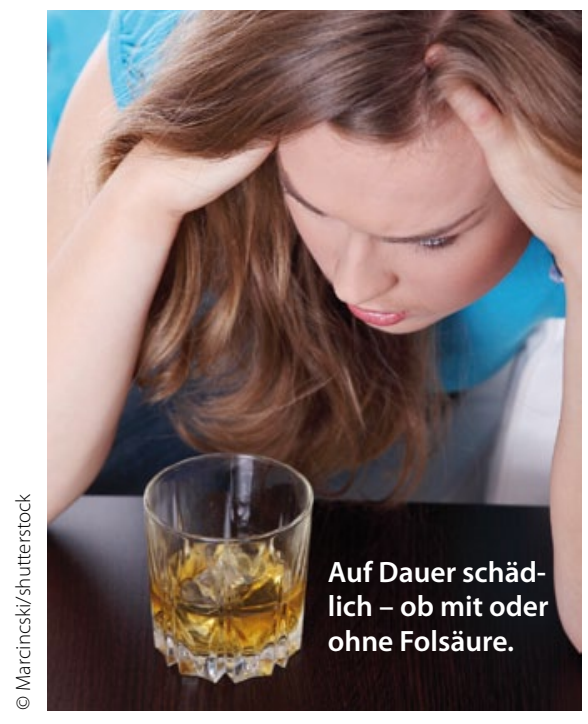

Die Auswertung bestätigte eine dosisabhängige Assoziation zwischen Alkoholkonsum und gutartigen Brustveränderungen. Pro $10 \mathrm{~g}$ Alkohol (etwa ein Glas Wein), die täglich im Alter zwischen 18 und 22 Jahren konsumiert wurden, stieg die Wahrscheinlichkeit für Brustveränderungen um 15\%. Mit Folsäure ließ sich dieser Zusammenhang nicht beeinflussen: Die Risikoerhöhung pro $10 \mathrm{~g}$ Alkohol war in jeder der drei Gruppen mit unterschiedlicher Folsäureaufnahme gleich.

$\mathrm{PL}$.

Liu Y et al. Pediatrics 2012, published online April 7; doi: 10.1542/peds.2011-2601 